\title{
The Pursuit of Knowledge and the Problem of the Unconceived Alternatives*
}

\author{
Fabio Sterpetti ${ }^{\dagger}$ \\ fabio.sterpetti@uniroma1.it \\ Marta Bertolaso: \\ m.bertolaso@unicampus.it
}

\begin{abstract}
In the process of scientific discovery, knowledge ampliation is pursued by means of nondeductive inferences. When ampliative reasoning is performed, probabilities cannot be assigned objectively. One of the reasons is that we face the problem of the unconceived alternatives: we are unable to explore the space of all the possible alternatives to a given hypothesis, because we do not know how this space is shaped. So, if we want to adequately account for the process of knowledge ampliation, we need to develop an account of the process of scientific discovery which is not exclusively based on probability calculus. We argue that the analytic view of the method of science advocated by Cellucci is interestingly suited to this goal, since it rests on the concept of plausibility. In this perspective, in order to account for how probabilities are in fact assigned in uncertain contexts and knowledge ampliation is really pursued, we have to take into account plausibility-based considerations.
\end{abstract}

\section{KEYWORDS:}

Analytic view of the method of science; Knowledge ampliation; Plausibility; Probability; Problem of the unconceived alternatives; Scientific discovery; Uncertainty.

* The article was jointly developed by both authors and the thesis commonly shared. Fabio Sterpetti was mainly responsible for the writing of sections 1, 3, 5, 7, and 7.1, Marta Bertolaso was mainly responsible for the writing of sections 2, 4, 6, 7.2, and 8 .

$\dagger$ Campus Bio-Medico University of Rome; Sapienza University of Rome.

* Campus Bio-Medico University of Rome. 


\section{Introduction: Knowledge and Uncertainty.}

If we pursue new knowledge, it means that we are dealing with an uncertain domain. If there were no uncertainty, no additional knowledge would be required to deal with that domain. When we deal with uncertainty, we usually resort to probability-based considerations, and try to shift from a condition of 'uncertainty' to one of 'risk', i.e. a condition in which a reliable estimation of the probability of each possible outcome is available. In a sense, "knowledge about risk" and uncertainty "is knowledge about lack of knowledge," and this "combination of knowledge and lack thereof contributes to making issues of risk" and uncertainty "complicated from an epistemological point of view" (Hansson 2014, Sect. 2). Indeed, probability cannot be assigned objectively to any possible event in a given domain unless we have knowledge about that domain which is certain. ${ }^{1}$ If we have reasons to think that our knowledge of a given domain is not certain, we try to acquire more knowledge about that domain in order to make probability assignment more objective. Acquiring more knowledge on a given domain and solving problems is precisely what scientific discovery aims at. In the pursuit of scientific knowledge, we perform ampliative reasoning, i.e. non-deductive reasoning. When ampliative reasoning is performed, probabilities cannot be

1 'Uncertainty' has different usages, at least an informal and a technical usage, and this may give rise to some misunderstandings. In order to make clearer what we mean by 'uncertainty', it may be useful to compare this concept with the concept of 'risk'. As Hansson states, in decision theory, "a decision is said to be made 'under risk' if the relevant probabilities are available and 'under uncertainty' if they are unavailable or only partially available. [...]. Although this distinction between risk and uncertainty is decision-theoretically useful," it is not completely satisfactory from an epistemological point of view, since "only very rarely are probabilities known with certainty. Strictly speaking, the only clear-cut cases of 'risk' (known probabilities) seem to be idealized textbook cases that refer to devices such as dice or coins that are supposed to be known with certainty to be fair. In real-life situations, even if we act upon a determinate probability estimate, we are not fully certain that this estimate is exactly correct, hence there is uncertainty" (Hansson 2014, Sect. 2). In this view, one deals with 'uncertainty' whenever one cannot make any probability estimate of the possible outcomes or one cannot know with certainty whether one's probability estimates are correct. 
assigned objectively (Bertolaso, Sterpetti 2017). One of the reasons is that we face the problem of the unconceived alternatives (Sklar 1981; Stanford 2006). We are unable to explore the space of all the possible alternatives to a given hypothesis. This is not accidental, nor is it limited to certain specific contexts (Rowbottom 2016). If we have reason to suspect that we are unable to claim that there cannot be unconceived alternatives to a given hypothesis $h$, we cannot affirm that $h$ is (approximately) true. In uncertain contexts, we routinely have reason to think that we do not know whether we are able to explore all the possible alternatives to $h$. When we try to extend our scientific knowledge beyond its current frontiers, we usually deal with uncertain contexts. And, indeed, we usually maintain that our scientific knowledge is fallible and revisable. If we were really able to explore all the possible alternatives to a given hypothesis $h$, we would have been able to find among them the 'best' one, and we could have been confident that this best hypothesis is (approximately) true, i.e. it corresponds to the way the world really is. If this were the case, our scientific knowledge would have been almost certain. But certainty does not admit of revision, since, as with knowing that $p$, being certain that $p$ entails that it is true that $p$. So, if our knowledge has to be seriously regarded as fallible (i.e. knowledge that is less than certain) and revisable, we have to assume that we are usually unable to claim that there cannot be unconceived alternatives to our favorite hypothesis. ${ }^{2}$ The same considerations apply to the process of probability assignment. In an uncertain context, if probabilities cannot be assigned in an objective way, each probability estimation amounts to a hypothesis that we formulate about the probability of a given case. So, if we want to adequately account for the process of knowledge ampliation in science and keep maintaining the idea that scientific knowledge is fallible and revisable, we need to develop an account of the process of scientific discovery which is not exclusively based on probability calculus. In order to account for how probabilities are in fact assigned in uncertain contexts, and knowledge ampliation and revision are really pursued, we have to take into account plausibility-based considerations (Cellucci $2017 ; 2013)$. In the rest of the article, we will try to adequately unpack the

2 On certainty and the difficulty of giving an account of certainty which is compatible with a fallibilistic view of knowledge, see Reed 2011, especially Sect. 2. 
argument sketched in this section and defend the several claims that figure in it which may appear disputable to many readers.

\section{The Search for a Logic of Discovery}

According to many authors, while there can be a logic of confirmation, since confirmation can be formalized, there cannot be a logic of discovery, since discovery processes cannot instead be formalized (Schickore 2014; Cellucci 2017). For example, Popper states that "there is no such thing as a logical method of having new ideas, or a logical reconstruction of this process" (Popper 2005, p. 8). The problem with this view is that it equates the intelligibility of a given reasoning process with the possibility of formalizing that process, i.e. the possibility of making that process algorithmically reproducible, and thus mechanizable. This approach leaves out from the perimeter of rational analysis and understanding both (1) the inferential paths of discovery that are not algorithmically describable, the ones on which we will focus in this paper (e.g. hypotheses production through nondeductive inferences); and (2) the non-algorithmic elements that contribute to those processes which, as confirmation, are thought to be algorithmically describable (think of the indispensable role that emotional circuits and subconscious inferences play in making us able to experience the 'sense of certainty' that we associate with valid deductive reasonings, see Rigo-Lemini, Martínez-Navarro 2017). But the fact that those elements cannot be formalized does not mean that they are irrational, nor that they cannot be analyzed at all.

Moreover, the asymmetry between discovery and confirmation is unjustified. As Putnam states, if we follow Popper and claim that there is no logic of discovery, because observations do not lead to theories "in a mechanical or algorithmic sense," then, "in that sense, there is no logic of testing, either" (Putnam 1975, p. 268). The idea that there can be a logic of confirmation because confirmation can be described in purely deductive terms, and so there can be an algorithmic method for confirmation, is unjustified. ${ }^{3}$ Algorithms are not able to account for all that is

3 Cf. Cellucci 2017, p. 142: "Methods can be divided into algorithmic and heuristic. An algorithmic method is a method that guarantees to always produce a correct solution to 
relevant to the process of hypotheses confirmation. Just as "there is no algorithmic method of discovery, there is no algorithmic method of testing. Indeed, by the undecidability theorem, there is not even an algorithmic method for testing whether a formula is logically valid or not" (Cellucci 2017, p. 144). ${ }^{4}$ So, either one admits that there cannot even be a logic of confirmation, or one should accept the idea that there can also be a logic of discovery.

In the last decades, the idea of developing a logic of discovery has been mainly intended as the attempt to develop a logic of inductive inferences in terms of probability calculus (Howson, Urbach 2006). ${ }^{5}$ The main problem with this approach is that it is mainly aimed at showing the validity and consistency of probabilistic inferences in the face of classical deductive logic. ${ }^{6}$ But in so doing, the probabilistic view of the logic of discovery becomes in a sense analogous to the deductivist view: it cannot take into account (and say something relevant about) some characteristic features of the process of discovery, namely how we produce and appraise new hypotheses. Those features cannot be straightforwardly

a problem. Conversely, a heuristic method is a method that does not guarantee to always produce a correct solution to a problem."

4 For a detailed explanation of why mathematical logic failed to be the logic of justification, see Cellucci 2015.

5 Here we cannot adequately deal with the heuristic approach to the logic of discovery for reasons of space, see Nickles 1980, 2009; Ippoliti 2015, forthcoming.

6 Cf. e.g. Howson, Urbach 2006, p. 79: “A nontrivial conclusion (one which is not itself a theorem of logic) of a deductively valid inference depends on at least one nontrivial premise. Similarly, a nontrivial conclusion (one which is not a theorem of probability) of a valid probabilistic argument depends on one or more nontrivial probabilistic premises. And just as the logical axioms in Hilbert-style axiomatisations of classical logic are regarded as empty of factual content because they are universally valid, so is the same true of the probability axioms in the view we have been advocating. They too are logical axioms, empty of factual content because universally valid. Putting all this together, we can derive a probabilistic analogue of the celebrated conservation result of deductive logic, that valid deductive inference does not beget new factual content, but merely transforms or diminishes the content already existing in the premises. So too here: valid probabilistic inference does not beget new content, merely transforming or diminishing it in the passage from premises to conclusion." 
formalized, nor can they adequately be described in probabilistic terms (more on this below). In this view, hypotheses production is just taken as a datum, something prior and external to a logic of discovery, precisely in the same way the process of hypotheses production is regarded as external to a logic of confirmation by those who deny that there can be a logic of discovery. ${ }^{7}$

\section{Ampliativity and Non-Objectivity}

There is a deep relation between (a) the non-deductive nature of ampliative reasoning and (b) the impossibility of assigning truly objective probabilities. In order to illustrate this point, consider some of the consequences (A and B) which can be drawn if we assume the following claims, which negate (a) and (b), respectively: (c) knowledge ampliation can be pursued by merely deductive methods; and (d) probability can be objectively assigned.

A) If it were possible to produce new hypotheses through merely deductive methods, and assign to these hypotheses an objective probability value, then our methods could be regarded as algorithmic methods and could be mechanized. So, discovery would be a routine and trivial task. Indeed, there is an algorithm to derive all deducible consequences from given premises (Cellucci 2017, Chap. 11).

B) In addition, our knowledge would be almost certain. If probabilities are really objective, in the sense that they are dictated to us by the way the world really is, they allow us to pick out the 'right' one among rival hypotheses through eliminative reasoning. If probabilities are dictated to us by the way the world really is, and we search for the probability that a given hypothesis is true, this means that probability can be regarded as a reliable indicator of truth, and so that the most probable hypothesis among a set of rival hypotheses can be regarded as true. ${ }^{8}$

7 By underlying these points, we do not mean to deny the theoretical relevance of both formal approaches to confirmation and probabilistic approaches to discovery. We only mean to stress that there may be some relevant theoretical insights in considering the role played by non-formalizable components of reasoning.

8 We are dealing here with an inference to the best explanation (Lipton 2004; see below, Sect. 7.2). In this kind of inference, some criterion is needed in order to rank different 
Please, note that it cannot be objected that if some correct hypothesis has not yet been formulated, this prevents us to reach certain knowledge, and even of being aware of it. This cannot be the case under the assumption we made, namely that probability is objective: if probability has to be regarded as really objective, then we can know for certain that there cannot be unconceived alternatives to the hypothesis we are evaluating. Otherwise, probabilities cannot be said to be really objective (more on this below). ${ }^{9}$ If we now apply deductive reasoning, which is truth-preserving, to the true hypotheses that we have been able to select in virtue of

explanations and select the one that displays the highest score, i.e. the best explanation. Here we consider the case that probability is an adequate criterion to rank rival hypotheses. In this case, the best hypothesis is the one which displays the highest score with respect to probability, and so the most probable hypothesis is regarded as the true one. We will not enter here the debate over the validity of the inference to the best explanation, nor the debate over the difficulty of ranking different hypotheses which are able to explain the same set of empirical phenomena but differ under some other theoretical respect (see e.g. Tulodziecki 2012). We just wish to stress that we are not focusing here on the epistemic and pragmatic difficulties that one has to face when one attempts to effectively determine the score of each hypothesis. We concede, for argument's sake, that hypotheses can be effectively ranked, for instance by performing reliable empirical tests. Rather, here we wish to focus on and appraise the theoretical consequences of accepting the claim that probability is objective, and it can be used to rank hypotheses. We thank an anonymous reviewer for pressing us to clarify this point.

9 This issue is strictly related to the 'bad lot argument' developed by van Fraassen (1989) against the inference to the best explanation. According to van Fraassen, we "can watch no contest of the theories we have so painfully struggled to formulate, with those no one has proposed. So our selection may well be the best of a bad lot. [...]. For me to take it that the best of set $\mathrm{X}$ will be more likely than not, requires a prior belief that the truth is already more likely to be found in X, than not" (van Fraassen 1989, p. 143). This objection is analogous to the 'problem of the unconceived alternatives' raised by Stanford (2006) against scientific realism. In both cases, the point is that we cannot exclude that there may be unconceived alternatives to the hypotheses we are evaluating. If probability is objective, probability values are assigned to each possible outcome, no one excluded, so it can be claimed that our 'lot' of hypotheses is not a bad lot, since no hypothesis is left out. In this case, we can claim that there cannot be unconceived alternatives, and the true hypothesis is among our set of hypotheses. 
the objectivity of their probabilities, we cannot go wrong in the process of knowledge ampliation, and our knowledge will keep being certain.

But A) and B) are highly implausible, since there is a wide consensus that: $A^{*}$ ) knowledge ampliation is not a routine, nor a trivial task; and $\mathrm{B}^{*}$ ) scientific knowledge is not certain, rather it is fallible and revisable. We will not discuss here whether it is true that scientific knowledge is fallible and revisable, nor whether it is true that knowledge ampliation is not a routine task, we are just interested in underlining that if scientific knowledge is regarded as fallible and knowledge ampliation as a non-routine task, then (c) and (d) cannot be held.

Moreover, if scientific knowledge can be ampliated by applying deductive methods to some given premises already established, given that deduction is nonampliative, this would amount to saying that all the knowledge that is derivable by those premises is in some sense already contained in those premises. ${ }^{10}$ But this view is not able to account for all those cases of knowledge ampliation in which our already established scientific knowledge is insufficient to solve a problem, and so new hypotheses (i.e. premises) need to be introduced. For example, when Cantor demonstrated that to every transfinite cardinal "there exist still greater cardinals, he

10 It may be objected that deductive arguments are at least ampliative in an epistemic sense, because otherwise we should say that we learn nothing in mathematics beyond what we already knew by knowing the premises of a proof. We agree that mathematical proofs can be regarded as ampliative in this epistemic sense, since we are not deductively omniscient. But epistemic ampliation is not equivalent to knowledge ampliation (see Cellucci 2017, Sect. 12.7), and here we are focusing on the latter. If mathematical proofs rest exclusively on deductions, a mathematical theorem "asserts nothing that is objectively or theoretically new as compared with the postulates from which it is derived, although its content may well be psychologically new in the sense that we were not aware of its being implicitly contained in the postulates" (Hempel 1945, p. 9). Deduction is extremely useful because it "discloses what assertions are concealed in a given set of premises, and it makes us realize to what we committed ourselves in accepting those premises." Nevertheless, "none of the results obtained by this technique ever goes by one iota beyond the information already contained in the initial assumptions" (Ibidem). So, if deduction is ampliative in a merely epistemic sense, it is unable to account for how we reach mathematical knowledge which cannot even in principle be deductively derived from what we already know. 
did not deduce this result from truths already known [...], because it could not be demonstrated within the bounds of traditional mathematics. Demonstrating it required formulating new concepts and new hypotheses about them" (Cellucci 2017, p. 310). This means that even in the case of mathematical knowledge, which is usually regarded as the paradigm of certain knowledge, new knowledge is not acquired by merely deductive methods from already established results. A fortiori, these considerations apply to the case of natural sciences. So, deductive methods are insufficient to account for knowledge ampliation. We resort to ampliative methods in the process of knowledge ampliation. But while deductive methods are truth-preserving, ampliative methods, which are non-deductive, are not truthpreserving. This implies that the results we arrive at by means of ampliative methods cannot be claimed to be true with certainty, even if their premises were known to be true for certain. Thus, those results shouldn't allow us to assign objective probability values. But if probability has to be objective, we should regard the results we arrive at by means of ampliative methods as true and certain. This is so implausible, not to say unacceptable, that let us suspect that something is wrong with assumptions (c) and (d).

The point is the following: if knowledge is fallible, fallibility has to enter the stage at some point. Now, if we assume that (c) knowledge ampliation can be pursued by merely deductive methods and (d) probability can be objectively assigned, there cannot be room for fallibility. So, we have to dismiss at least one of our assumptions. The crucial one is (d). Indeed, that deductive methods are truthpreserving is undisputed. So, deductive methods cannot make room for fallibility unless their premises are not certainly true. But their premises cannot be but certainly true if probability is objective, because, as already noted, if probability were really objective, we would be able to select the true hypothesis among its rivals, independently from whether we arrive at such hypothesis through deductive or non-deductive methods. So, probability cannot be regarded as really objective, and we have to dismiss (d).

Now, consider what happens if we dismiss (d). We obtain a more familiar view of scientific knowledge. If probability is not really objective, we cannot know whether the premises of deductive reasoning are true, so the results established by deductive methods are now possibly false. The results we arrive at by ampliative methods are fallible as well, because, as already noted, ampliative methods are not 
truth-preserving. So, all our knowledge is fallible, and thus revisable. This also means that objective probability and infallible knowledge are unattainable. Indeed, if neither the results established by deductive methods, nor the results established by ampliative methods can be known to be true for certain, we cannot know whether the probability values we assign in the domain we are investigating correspond to objective probabilities. And if we cannot know whether the probability values we assign correspond to objective probabilities, we cannot know whether the results we arrive at by deductive and ampliative methods are true, and so on.

As we have seen, there is a deep relation between the non-deductive nature of ampliative reasoning and the impossibility of assigning truly objective probabilities. So, in order to develop an adequate account of the process of knowledge ampliation, at least two elements have to be added to the traditional view of logic: $\alpha$ ) an adequate treatment of ampliative reasoning; and $\beta$ ) an adequate treatment of the issue of probability assignment. According to the view advocated here, in both $\alpha$ ) and $\beta$ ) a pivotal role is played by the concept of plausibility.

\section{Knowledge Ampliation and Plausibility}

An interesting proposal aimed at modeling the process of knowledge ampliation is the analytic view of the method of science (Cellucci 2013, 2016, 2017), according to which knowledge is increased through the analytic method. In this view, "to solve a problem one looks for some hypothesis that is a sufficient condition for solving it. The hypothesis is obtained from the problem, and possibly other data already available, by some non-deductive rule, and must be plausible [...]. But the hypothesis is in its turn a problem that must be solved, and is solved in the same way" (Cellucci 2013, p. 55). ${ }^{11}$

Assessing the plausibility of any given hypothesis is crucial in this perspective. But how plausibility has to be understood? The interesting suggestion made by the

11 The origin of the analytic method may be traced back to the works of the mathematician Hippocrates of Chios and the physician Hippocrates of Cos, and was firstly explicitly formulated by Plato in Meno, Phaedo and the Republic. See Cellucci 2017; 2013. 
analytic view is that, in the ultimate analysis, the plausibility of a hypothesis is assessed by a careful examination of the arguments (or reasons) for and against it. ${ }^{12}$

According to this view, in order to judge over the plausibility of a hypothesis, the following 'plausibility test procedure' has to be performed: (1) "deduce conclusions from the hypothesis"; (2) "compare the conclusions with each other, in order to see that the hypothesis does not lead to contradictions"; (3) "compare the conclusions with other hypotheses already known to be plausible, and with results of observations or experiments, in order to see that the arguments for the hypothesis are stronger than those against it on the basis of experience" (Ibidem, p. 56). If a hypothesis passes the plausibility test procedure, it can be temporarily accepted. If, on the contrary, a hypothesis does not pass the plausibility test, it is put on a 'waiting list', since new data may always emerge, and a discarded hypothesis may successively be re-evaluated. ${ }^{13}$ Thus, according to the analytic view of method, what in the ultimate analysis we really do, in the process of knowledge ampliation, is producing hypotheses, assessing the arguments/reasons for and the arguments/reasons against each hypothesis, and provisionally accept or refute such hypotheses.

\section{Plausibility and Probability}

It is important to stress the difference between the concept of probability and the concept of plausibility. As Kant points out, "plausibility is concerned with whether, in the cognition, there are more grounds for the thing than against it" (Kant 1992, p. 331), while probability measures the relation between the winning cases and

12 On the concept of 'plausibility', see also Gettys, Fisher 1979; Agassi 2014. These views of plausibility differ from Cellucci's view under several respects. Nevertheless, all these conceptions of plausibility share some common features, namely the idea that plausibility assessment cannot be reduced to probability calculus, and that plausibility assessment is crucial for ampliating knowledge.

13 For a more detailed treatment of this issue, see Cellucci 2017, Chap. 9, where plausibility is confronted with related (but distinct) concepts, such as truth, probability, and warranted assertibility. 
possible cases. Plausibility involves a comparison between the arguments for and the arguments against, so it is not a mathematical concept. Conversely, probability is a mathematical concept (see Cellucci 2013, Sect. 4.4).

It may be objected that, although probability and plausibility appear to be distinct concepts, we may account for plausibility-based considerations in terms of probability, because plausibility obeys the law of probability (Pólya 1941). But this objection is inadequate. To see that plausibility is not equivalent to probability, consider that, since probability is "a fraction whose numerator is the number of favorable cases and whose denominator is the number of all the cases possible" (Laplace 1951, p. 7), in order to effectively calculate the probability of a given hypothesis $h$, we have to know the denominator, i.e. the number of all the cases possible. But in many cases, we do not know (and perhaps we cannot even know) the number of all the cases possible. Thus, if plausibility were to be understood in terms of probability, we could not be able to evaluate the plausibility of all those hypotheses for which we are unable to determine the set of all the possible rival alternatives. But we routinely evaluate the plausibility of that kind of hypotheses, so it cannot be the case that probability is equivalent to plausibility.

Moreover, that plausibility has to be distinguished from probability clearly appears by considering the fact that there are hypotheses that are plausible, but which, according to probability theory, have zero probability, while there are hypotheses that are highly implausible, but which, according to probability theory, have non-zero probability. ${ }^{14}$ Thus, contrary to Pólya (1941), we should conclude

14 See Cellucci 2013, Chap. 20. An example of plausible hypotheses that have zero probability are all the plausible hypotheses derived by an Induction from a Single Case (ISC). On the classical concept of probability as the ratio between favorable and possible cases, a conclusion obtained by (ISC) has zero probability when the number of possible cases is infinite. An example of implausible hypotheses that have non-zero probability are those implausible hypotheses obtained by Induction from Multiple Cases (IMC). Consider the hypothesis that all swans are white. Until the end of the seventeenth century, "all swans observed were white. From this, by (IMC), it was inferred that all swans are white. But in 1697 black swans were discovered in Western Australia." Since then, the hypothesis that all swans are white is highly implausible. But, this contrasts with the fact that, "on the classical concept of probability, a conclusion obtained by (IMC) has non-zero probability when the number of possible 
that the 'calculus of plausibilities' does not obey the rules of the calculus of probabilities, and that plausibility has to be distinguished from probability.

Finally, that plausibility has to be distinguished from probability can also be seen by considering how we estimate the degree of uncertainty of the way we estimate the degree of uncertainty of a given event. For example, according to Calude and Longo (2106), randomness is "unpredictability with respect to the intended theory and measurement" (p. 266). In this view, probability is a measurement of randomness, and randomness is a kind of unpredictability which derives from the theoretical assumptions one makes. So, the probability values that one assigns to the set of the possible outcomes of a given domain $\mathrm{A}$ are dependent on one's theoretical assumptions. This means that in order to assign probability values, one has to previously make a theoretical choice. Since the choice of the theoretical framework one decides to deal with is indispensable in order to assign probability values in the A-domain, this choice cannot in its turn be made by relying on probability-based considerations. Otherwise a regression is lurking. Indeed, if one commits oneself to a given theoretical framework, say $T_{a}$, in order to assign probability values in the A-domain, and, if in order to pick out $T_{a}$ from the set $\mathrm{T}$ of similar but not equivalent theoretical frameworks, i.e. $T_{a}, T_{a} *, T_{a}{ }^{* *}$, etc., one relies on probability-based considerations, this means that one can assign a probability value to each member of T, i.e. $T_{a}, T_{a} * T_{a}{ }^{* *}$, etc. And one can do that because one has already chosen a theoretical framework of a higher order, say $F_{t}$, which allows one to assign probability values in the $\mathrm{T}$ domain. Now one has to account for how to choose $F_{t}$ among similar but not equivalent theories in set $\mathrm{F}$. And so on. Thus, in order to avoid a regress, at a certain point we have to account for the way one chooses the theoretical framework which allows one to assign probability values in the domain of interest in some different way, i.e. without relying on probability-based considerations. This choice, we claim, is made by relying on plausibility-based considerations. Indeed, reasons and arguments that support different theoretical frameworks can be assessed even if we are unable to coherently assign probability values to rival theoretical frameworks.

cases is not infinite," and such "is the case of the hypothesis that all swans are white" (Cellucci 2013, p. 335). 
As an example, consider the case of the "uncertainty about probabilities" (see Teira 2011, Sect. 6). Statisticians may well estimate the degree of uncertainty in their dealing with uncertainty. For instance, they can assign a probability $p^{*}$ to the hypothesis that their assignment of probability $p$ to event $e$ in a given domain $\mathrm{D}$ is correct. If we want to stop the regress, we need to account for the process of evaluation of the method through which statisticians assign both those probabilities, i.e. $p^{*}$ and $p$, in terms of plausibility.

Consider now the possibility of knowing the space of all possible alternatives to a given hypothesis. If it were possible to know the space of all possible alternatives to a given hypothesis $h$, we could test all of them and assign objective probabilities to each alternative hypothesis $h_{i}$. This would amount to saying that the procedure of hypotheses evaluation can always be performed through an algorithmic method and can be mechanized. Indeed, we can develop an algorithm that enumerates all the possible alternative hypotheses to a given hypothesis $h$, assigns to each of them the relative objective probability, calculates the likelihood of each hypothesis, and then picks out the one which displays the higher likelihood. This would render the process of hypotheses evaluation a trivial task. But, since we usually do not know the space of all possible alternatives to a given hypothesis $h$, we have instead to rely on plausibility-based considerations in order to assess $h$. This shows that there is a deep relation between the need of taking into account plausibility-based considerations in the attempt to develop a logic of discovery and the impossibility of knowing the space of all the possible alternatives to a given hypothesis.

\section{Plausibility and Rationality.}

It may be objected that if one deals with plausibility-based considerations, one risks being irrational, because one is prone to violate the laws of probability, given that plausibility does not follow the laws of probability calculus. To face this objection, we will stick to Pollock's defense from the same objection. Pollock (1983) argues that it is impossible to equate what he calls 'epistemic probability' and 'statistical probability'. It has to be noted that his conception of 'epistemic probability' is more akin to what we call 'plausibility' than to what is usually understood by the term 'probability'. According to Pollock, 'statistical probability' 
is that kind of probability "about which we can learn by discovering relative frequencies, counting cases" (Pollock 1983, p. 236). On the contrary, the "epistemic probability of a proposition is the degree to which it is warranted," but it is "not immediately obvious whether epistemic probability can be quantified, and even if it can it is far from obvious that it will satisfy the probability calculus" (Ibidem). In this view, a proposition is deemed warranted by a careful examination of the reasons for and against it: "a person is justified in believing P just in case he has adequate reason to believe P [...], and he does not have any defeaters for it at his immediate disposal" (p. 233). Thus, the evaluation of the "epistemic probability' of a belief/proposition rests on the evaluation of the strength of the arguments that justify that belief/proposition. Pollock clearly denies that arguments' evaluation can be represented in probabilistic terms. Indeed, he also explicitly denies the possibility of equating 'epistemic probability' with 'subjective probability', i.e. probability assigned in Bayesian fashion. In his view, even if we follow the Bayesians and regard probability as expressing a person's 'degree of belief' in the truth of a certain proposition, we cannot equate probability assignment with arguments evaluation, mainly because we cannot reasonably impose on arguments evaluation the rules of probability calculus.

The main argument used by the Bayesians to maintain that our estimation of epistemic probability must obey the rules of probability calculus is a rationality requirement. They take our degree of belief to be equivalent to our disposition to bet on the truth of a proposition. So, if "when presented with a set of propositions and required to bet on the likelihood of their being true a person did not compute his odds in accordance with the probability calculus, then a wily opponent could place a set of bets which the person would be bound to lose no matter what" (p. 244). Thus, the argument goes, since if we violate probability calculus in our assignment of prior probabilities, we will be prone to Dutch books, and so our behavior will be irrational, priors assignment has to conform to the rules of probability. ${ }^{15}$ Pollock finds this argument unconvincing, because the "question of how good are one's epistemic reasons for believing something is quite different from the question whether it is to one's advantage to believe it, and that in turn is

15 On the relevance of the Dutch Books Arguments in the debate over the rationality requirement advocated by the Bayesians, see Vineberg 2016. 
different from the question whether it is to one's advantage to bet on it [...]. Thus," he does "not think that the Dutch book argument can be regarded as establishing that epistemic probabilities must conform to the probability calculus" (p. 244). In other words, according to Pollock, there are two distinct epistemic aims that may well diverge: 1) our desire to avoid irrational behavior, which leads us to conform to probability calculus in priors assignment, and 2) our desire to assign priors on the basis of our rational assessment of the reasons that we actually have found so far to justify them, which may lead us to violate the rules of probability. Whether we decide to be rational in the sense of conforming to probability calculus or in the sense of following our assessment of reasons may depend on the context and ultimately rests on an epistemic choice that we have to make. And the way we make this choice, we argue, cannot be accounted for in terms of probability, rather it is better accounted for in terms of plausibility. So, in Pollock's view, epistemic probability and statistical probability may diverge, and epistemic probability does not conform to probability calculus. Even if we adopt a Bayesian framework, epistemic probability cannot be made coincident with Bayesian's 'subjective probability', i.e. the degree of probability that we estimate. The point is that "when we assess the degree of warrant of the conclusion of an argument, we do not somehow combine all of these considerations in some fancy mathematical calculation to arrive at a computed degree of warrant for the conclusion" (p. 249). Pollock seems to maintain the idea that 'statistical probability' and 'subjective probability' are mathematical concepts, while 'epistemic probability' is not a mathematical concept. It seems quite fair to say that what Pollock calls 'epistemic probability' is not, despite its name, really a kind of probability, but it is a concept very similar to what we refer to as 'plausibility'. If we speak, for clarity, of 'plausibility' instead of 'epistemic probability', what Pollock is saying is that if one deals with plausibility, the charge of being irrational because one does not conform to probability calculus is simply misdirected, because it mistakes a nonmathematical concept, i.e. plausibility, for a mathematical one. Since plausibility is not a mathematical concept, the process of plausibility estimation cannot conform to the laws of probability calculus, which are mathematical in character. So, it cannot be said that if one deals with plausibility estimation one is prone to violate the laws of probability, and so one risks being irrational. Thus, this objection is inadequate. 


\section{Probability and the Problem of the Unconceived Alternatives}

We now wish to elaborate further on two related claims we made above, namely (1) knowing the space of all possible alternatives to a given hypothesis $h$ is necessary in order to assign objective probability to $h$, and (2) if probability is objective, there cannot be unconceived alternatives to $h$. Consider a standard sixfaces die. We know that there are precisely six possible outcomes for one throw of that die. The space of possibilities is completely determined in advance by the symmetries of the system under investigation, and this allows us to safely assign probabilities to possible outcomes. These probabilities can be regarded as objective (despite some abstractions and idealizations may be required if we are dealing with a real die). That these probabilities are objective implies that there cannot be other possible outcomes we failed to consider. So, we are confident that those probability values will never be modified. But usually in science, when we try to solve a problem, we are not in such a position. We do not know in advance the space of possibilities for the phenomenon we want to explain. Nor do we know the exact configuration of the space of all the possible alternative hypotheses that may be formulated in order to explain that phenomenon. If the space of possible theoretical alternatives to a given hypothesis $h$ is not determinable in advance, we cannot safely claim to have exhaustively searched that space, found that $h$ is the hypothesis that best explains the phenomenon under investigation, and so that we should trust $h$ because it is confirmed by the eliminative inferential procedure we performed. This is the problem of the unconceived alternatives. ${ }^{16}$

16 It is worth clarifying that we are not claiming that one should not rely on estimated probabilities for practical purposes, nor are we denying that those who rely on estimated probabilities for practical purposes are successful in dealing with the world. Here we are focusing on the epistemological analysis of the process of scientific discovery, which is a distinct issue. That estimated probabilities may well be adequate for dealing with every-day activities does not impinge on the theoretical issue of assessing whether scientific knowledge is certain. Indeed, the consequences of not considering some unknown possibilities may well be negligible in some contexts. Humans built ships long before coming to understand on scientific grounds why they were able to build ships 
This problem has been in recent years fiercely stressed by Stanford (2006). ${ }^{17}$ The peculiarity of Stanford's defense of the instrumentalist attitude towards science, according to which we should refrain to commit ourselves to the existence of the theoretical entities posited by our best scientific theories, is that it shifts the focus of the debate over scientific realism from the theories to the theorists (Forber 2008; Saatsi et al. 2009). While traditional anti-realist arguments are based on the analysis of the historical record of theory change (e.g. Laudan 1981), Stanford's argument relies on the consideration that the historical record of science points out that we humans routinely failed to conceive all the possible alternatives to a given theoretical hypothesis $h$ at the time it was formulated, and that this prevents us to claim that any current theoretical hypothesis $h$ is true. Before Stanford's proposal, analogous concerns were made by van Fraassen in his criticism of the inference to the best explanation (van Fraassen 1989),${ }^{18}$ and by Sklar, who considered the role played by the unconceived alternatives in the case of the inference to the best explanation and in the case of confirmation theories (Sklar 1981). To better

that floated. Nevertheless, their practical ability in building ships did not make their prescientific beliefs about the reasons why they were able to build ships that floated more justified. The point is that practical success is not able to justify by itself our beliefs about the reasons why we succeeded in practical activities. As regard estimated probabilities, in many cases, even "if a decision problem is treated as a decision 'under risk', this does not mean that the decision in question is made under conditions of completely known probabilities. Rather, it means that a choice has been made to simplify the description of this decision problem by treating it as a case of known probabilities. This is often a highly useful idealization in decision theory" (Hansson 2014, Sect. 2). But the fact that we can deal with some practical affairs to a satisfactory extent by assuming that our probabilities are objective does not mean that our probabilities are really objective, and we really know for certain that there cannot be unconceived alternatives. We wish to thank an anonymous reviewer for urging us to clarify this point.

17 Many replies have been elaborated in the last decade to address Stanford's instrumentalist challenge to scientific realism. See e.g. Magnus 2006, 2010; Saatsi et al. 2009; Ruhmkorff 2011; Devitt 2011; see Saatsi et al. 2009 for Stanford's rejoinder to some criticisms; see Rowbottom 2016 and Wray 2016 for interesting extensions of Stanford's line of reasoning.

18 See above, fn. 9. 
illustrate how the problem of the unconceived alternatives is related to the issue of plausibility, in the rest of this section we discuss both these cases.

\subsection{Confirmation Theories}

Let us begin with the case of confirmation theories, which are usually developed in terms of probabilities. A clear formulation of the problem of the unconceived alternatives in this context can be found in Sklar (1981):

Consider Bayesian strategies for confirmation theory. Here we must distribute a priori probabilities over all the alternative hypotheses to be considered. If there is only a finite set of hypotheses we have in mind, this is easy to do [...]. But if we must keep in mind the infinite and indeterminate class of all possible hypotheses, known and unknown, how can we even begin to assign a priori probabilities to those few hypotheses [...] we do have in mind [...]? (Sklar 1981, p. 19).

We will follow (and simplify a bit) Rowbottom (2016) in order to better illustrate this point. In Bayesian theories of confirmation, the confirmation of a hypothesis $h$ is equal to its conditional probability given some evidence $e$ :

$$
\mathrm{P}(h, e)=\mathrm{P}(h) \mathrm{P}(e, h) / \mathrm{P}(e)
$$

where $\mathrm{P}(h, e)$ is the conditional probability, $\mathrm{P}(h)$ and $\mathrm{P}(e)$ are the prior probabilities respectively of $h$ and $e$, and $\mathrm{P}(e, h)$ is the likelihood. In this approach, the prior probability of $e$ must be determined considering all the alternatives to $h$. Indeed, $\mathrm{P}(e)$ decomposes as follows:

$$
\mathrm{P}(e)=\mathrm{P}(h) \mathrm{P}(e, h)+\mathrm{P}(\sim h) \mathrm{P}(e, \sim h)
$$

and $\mathrm{P}(\sim h) \mathrm{P}(e, \sim h)$ in its turn decomposes into:

$$
\mathrm{P}\left(\sim h_{1}\right) \mathrm{P}\left(e, \sim h_{l}\right)+\ldots+\mathrm{P}\left(\sim h_{n}\right) \mathrm{P}\left(e, \sim h_{n}\right)
$$


where the set of all the possible alternatives to $h$ is $\left\{\sim h_{1}, \ldots, \sim h_{n}\right\}$.

In this perspective, theories are considered to be highly confirmed provided that $\mathrm{P}(\sim h) \mathrm{P}(e, \sim h)$ is low, i.e. when the probability assigned to the negation of the proposed hypothesis is low. Confirmation theorists call $\sim h$ the 'catchall hypothesis', i.e. the hypothesis that incorporates all the alternatives to $h .^{19}$

To sum up, in order to confirm a hypothesis $h$, we have to assign $\mathrm{P}(e)$; in order to assign $\mathrm{P}(e)$, we have to be able to estimate $\mathrm{P}(e, \sim h)$; and in order to estimate $\mathrm{P}(e$, $\sim h$ ), we have to be able to construct the set of all the possible alternatives to $h$ and assign a prior probability to each of those alternatives.

The impossibility to construct the actual set of all the possible alternatives to a given theoretical hypothesis $h$ has been clearly stated by Salmon:

\begin{abstract}
At any given stage of scientific investigation, the catchall is the disjunction of all of the hypotheses we have not yet conceived. What is the likelihood of any given piece of evidence with respect to the catchall? This question strikes me as utterly intractable; to answer it we would have to predict the future course of the history of science. (Salmon 1990, p. 329).
\end{abstract}

Salmon's solution to the problem of the unconceived alternatives for confirmation theory is to consider, when evaluating the confirmation of a given hypothesis $h$, only the actually conceived alternatives to $h$. This is an example of a plausible theoretical choice, because it allows us to produce at least an estimation of the degree of confirmation of a given hypothesis, although a provisional and revisable one. But this choice cannot be justified in terms of probability. This theoretical choice can be evaluated and eventually accepted by pondering the

19 If rival hypotheses are not only assumed to be mutually exclusive but also jointly exhaustive, "then one's model will represent a situation in which one knows that one of these competing hypotheses must be true. In such a case, there is no need to include a 'catch-all' hypothesis to represent all unimagined hypotheses," but "there are many contexts in which it is not known with certainty that the true hypothesis is one of those considered" (Schupbach 2011, p. 119). The problem in these cases is that we cannot demonstrate that there cannot be other unimagined hypotheses. "Such scenarios correspond to van Fraassen's best of a bad lot objection as well as what Kyle Stanford (2006) calls “the problem of unconceived alternatives"' (Schupbach 2011, p. 119, fn. 2). 
arguments for and against it, i.e. by assessing its plausibility. Salmon's solution to the problem of the unconceived alternatives for confirmation theory is just an example of the fact that, in the process of knowledge ampliation, we cannot rely exclusively on probability-based considerations, we have to rely also on plausibility-based considerations. And the process by which we evaluate this kind of considerations cannot be made algorithmic, and so cannot be mechanized.

Moreover, as Rowbottom clearly points out, by adopting Salmon's move, we can effectively calculate the confirmation of a given hypothesis $h$. But now we are not dealing anymore with the calculation of an absolute value for the degree of confirmation of $h$ by the evidence $e$, rather we are dealing with the relative confirmation of $h$, i.e. the degree of confirmation of $h$ if we consider only the conceived alternatives. ${ }^{20}$ However, "relative confirmation has no established connection to truth-likeness, even on the assumption that absolute confirmation (in some non-subjective sense) does indicate truth-likeness" (Rowbottom 2016, p. 3). Thus, even if $\mathrm{P}(\sim h) \mathrm{P}(e, \sim h)$ is now low, i.e. $h$ is confirmed, it would become dramatically higher, if a new serious alternative to $h$ predicting $e$ became apparent. ${ }^{21}$ This possibility prevents us to straightforwardly claim that the probabilities we assigned to the conceived alternatives in order to confirm $h$ are objective. Indeed, the probabilities that we assign can be straightforwardly claimed to be objective only if we can reliably affirm to know the space of all the possible outcomes with certainty, i.e. if we can claim that there cannot be unconceived alternatives.

It is worth noticing that while the idea that the confirmation of a given hypothesis may vary if a new serious but so far unconceived alternative becomes apparent is in contrast with the idea that probability is objective, it perfectly

20 Cf. Stanford 2006, p. 42: "There is simply no way to assign an absolute probability or level of confirmation to the theory without solving the problem of estimating the likelihood conferred on the evidence by a catch-all hypothesis of unknown content and constitution."

21 Cf. Rowbottom 2016, p. 3: "Why be confident that the confirmation value of any given theory $[\ldots]$ would not change drastically if all the unconceived alternatives were appreciated? What licenses inferring absolute confirmation values from relative confirmation values?" 
squares with the analytic view of the method of science. In this view, the evaluation of a given hypothesis is not performed once for all. When a hypothesis passes the plausibility test procedure, it is temporarily accepted, because new data may always emerge, and a hypothesis may always be re-evaluated (see above, Sect. $3)$.

\subsection{Inference to the Best Explanation}

As regard the inference to the best explanation, this inference is prone to analogous criticisms ${ }^{22}$ which rest on the impossibility of determining the space of the possible alternatives to a given hypothesis:

Consider inference to the best explanation. Should we adopt that hypothesis relative to which the evidence has the highest likelihood of all the likelihoods generated by the hypotheses we have in mind as alternatives? Rather, shouldn't we realize that in the vast sea of alternatives we have not yet considered it is all too probable that there is some, as yet inconceived, hypothesis relative to which the evidential warrant is even better explained than it is by our current best candidate? (Sklar 1981, p. 19).

To better see the relation between the inference to the best explanation (an inference rule introduced by Harman (1965) but already considered by Peirce, see e.g. Kapitan 1992), and Bayesian approaches to confirmation, consider that when one performs an inference to the best explanation, "one infers, from the premise that a given hypothesis would provide a 'better' explanation for the evidence than would any other hypothesis, to the conclusion that the given hypothesis is true" (Harman 1965, p. 89). So, both confirmation theories and the inference to the best explanation can be regarded as instances of eliminative reasoning. They both work by comparing some rival items of a given set (i.e. hypotheses to be confirmed, explanations to be evaluated) with each other and then by selecting the one which displays the highest score with respect to a given parameter. And so, they both have to face the problem of the unconceived alternatives. Indeed, in both cases, if

22 See above, fn. 9. 
the space of all the possible alternatives is not known, the outcome of such selective inferential procedures might be off-track. But the relation between the inference to the best explanation and Bayesian confirmation theories is even deeper. Indeed, many authors think that, if one deals with scientific issues, in order to define what a 'best' explanation is in an 'objective' way, one has to resort to probability-based considerations. In this view, one relies on Bayes' theorem to determine which is the best explanation. Howson, for instance, denies that, in order to determine which is the 'best' explanation among some rival scientific hypotheses, an appeal to what is scientifically plausible can help. In his view:

What is plausible and what is not is likely to be a highly context-dependent affair, if not an outright subjective one, unless it is tied to some objective standard. That of probability naturally comes to mind. There is nothing in principle wrong with combining the standard of inference to the best explanation with an explicitly probabilistic criterion of 'best': you simply 'abduce' to the most probable among a pre-assigned set of alternative explanatory hypotheses, if there is a uniquely a posteriori most probable one. (Howson 2000, p. 112).

But, as noted above, if we rely on Bayes' theorem to determine the most probable hypothesis in an objective way, the question is just begged, since we now have to face the problem of assigning a prior probability value to each possible alternative hypothesis. And prior probabilities cannot be usually assigned in an objective way because we do not even know how the space of all the possible rival alternatives is shaped, let alone how probable each hypothesis is. As Howson himself states "a posteriori probability depends on a priori probability, and a priori probability assignments seem to be irretrievably dependent on either an arbitrary or a subjective decision" (Ibidem).

The two cases briefly discussed (i.e. confirmation theories and the inference to the best explanations), show that when we try to extend our knowledge by performing ampliative reasoning, at least at some point in our reasoning there is not a real 'objective' alternative to assessing rival hypotheses on the basis of plausibility-based considerations. So, it seems fair to say that an account of scientific discovery exclusively cast in terms of probability-based considerations is unsatisfactory. In our view, we should acknowledge (and account for) the 
indispensable role played by plausibility-based considerations in (at least) those contexts in which we do not know in advance with certainty how the space of all the possible alternatives is shaped. ${ }^{23}$

\section{Conclusion: Subjectivity and Arbitrariness}

There is a sense in which the attempt to avoid subjectivity in pursuing knowledge has a positive meaning, namely when it means avoiding personal biases. For example, the adoption of systematic methodologies allowed clinical medicine to become a science, precisely because those methodologies allowed us to eliminate personal biases from medical practice (Bird 2017). But not every subjective element in the process of knowledge production can be regarded as a bias producer. The risk is that in some circumstances the quest for objectivity ends in simply hiding some of the subjective components of the processes of knowledge ampliation. ${ }^{24}$ We think that the concept of plausibility described above can be of help in the attempt to counter this tendency and untangle the notion of subjectivity from that of arbitrariness. Some authors seem to think that if knowledge is not objective (i.e. if it does not leave out any subjective element), then knowledge is arbitrary, and so there is no real knowledge at all. Contrary to this view, we argued that some subjective elements of reasoning cannot be eliminated, since the process of plausibility evaluation of the hypotheses cannot be made algorithmic, nor can it be ruled out. In this perspective, there would be no knowledge only if the hypotheses we deal with in the process of knowledge ampliation were arbitrary.

23 We say 'at least' because it can be argued that plausibility-based considerations play an indispensable role also in those cases in which the space of the possible alternatives is known with certainty, because the preliminary judgement that we do know the shape of the possible alternatives with certainty is better accounted for in terms of plausibilitybased considerations, rather than in terms of probability-based considerations. We have no space here to further develop this argument.

24 Cf. e.g. Howson, Urbach 2006, p. 9: "the ideal of total objectivity is unattainable and [...] classical methods, which pose as guardians of that ideal, actually violate it at every turn; virtually none of those methods can be applied without a generous helping of personal judgment and arbitrary assumption." 
But they need not be arbitrary, they must be plausible, i.e. the arguments for them have to be stronger than the arguments against them. If the plausibility evaluation of hypotheses is carefully conducted, even if this process cannot be formalized, it nevertheless cannot be regarded as arbitrary, since it is constrained in several rational ways. Thus, knowledge may well be possible, even if some subjective elements enter into the process of knowledge production. Better, knowledge is possible precisely because those subjective elements enter into the process of knowledge ampliation and allow us to deal with uncertain contexts.

To sum up, in this article we argued that: 1) not every aspect of reasoning is reducible to probability calculus, but this does not imply that those aspects which do not conform to the rules of probabilities are irrational; 2) the best way to account for such aspects of reasoning is by considering the role that plausibilitybased considerations play in our reasoning processes; 3 ) if one wishes to develop a satisfactory logic of discovery, one has to consider both 1) and 2).

\section{References}

Agassi, J. (2014). Proof, Probability or Plausibility. In: Mulligan, K., Kijania-Placek, K., Placek, T. (eds.), The History and Philosophy of Polish Logic. London: Palgrave Macmillan: 117-127.

Bertolaso, M., Sterpetti, F. (2017). Evidence Amalgamation, Plausibility, and Cancer Research. Synthese, DOI: 10.1007/s11229-017-1591-9.

Bird, A. (2017). Systematicity, Knowledge, and Bias. How Systematicity Made Clinical Medicine a Science. Synthese, DOI:10.1007/s11229-017-1342-y.

Bueno, O., Neiman, R. (2017). Partial Evidence in Medicine. In: Chao, H-K., Reiss, J. (eds.), Philosophy of Science in Practice: Nancy Cartwright and the Nature of Scientific Reasoning. Springer: Dordrecht: 25-44.

Calude, C., Longo, G. (2016). Classical, Quantum and Biological Randomness as Relative Unpredictability. Natural Computing, 15(2): 263-278.

Cellucci, C. (2013). Rethinking Logic. Logic in Relation to Mathematics, Evolution, and Method. Dordrecht: Springer.

Cellucci, C. (2015). Why Should the Logic of Discovery Be Revived? A Reappraisal. In: Ippoliti, E. (ed.), Heuristic Reasoning. Cham: Springer: 11-27. 
Cellucci, C. (2016). Models of Science and Models in Science. In: Ippoliti, E., Sterpetti, F., Nickles, T. (eds.), Models and Inferences in Science. Cham: Springer: 95-122.

Cellucci, C. (2017). Rethinking Knowledge. The Heuristic View. Dordrecht: Springer.

Devitt, M. (2011). Are Unconceived Alternatives a Problem for Scientific Realism? Journal for General Philosophy of Science, 42(2): 285-293.

Forber, P. (2008). Forever Beyond our Grasp? Review of P. Kyle Stanford (2006), Exceeding Our Grasp. Biology and Philosophy, 23(1): 135-141.

Gettys, C.F., Fisher, S.D. (1979). Hypothesis Plausibility and Hypothesis Generation. Organizational Behavior and Human Performance, 24(1): 93-110.

Hansson, S.O. (2014). Risk. In: Zalta, E.N. (ed.), The Stanford Encyclopedia of Philosophy, $\mathrm{URL}=\langle$ https://plato.stanford.edu/archives/spr2014/entries/risk/ $>$.

Harman, G.H. (1965). The Inference to the Best Explanation. The Philosophical Review, 74(1): 88-95.

Hempel, C.G. (1945). Geometry and Empirical Science. The American Mathematical Monthly, 52(1): 7-17.

Howson, C. (2000). Hume's Problem. Oxford: Oxford University Press.

Howson, C., Urbach, P. (2006). Scientific Reasoning. The Bayesian Approach. $3^{\text {rd }}$ edition. Chicago and La Salle: Open Court.

Ippoliti, E. (2015). Reasoning at the Frontier of Knowledge: Introductory Essay. In: Ippoliti, E. (ed.), Heuristic Reasoning. Cham: Springer: 1-10.

Ippoliti, E. (forthcoming). Heuristic Logic. A Kernel. In: Danks, D., Ippoliti, E. (eds.), Building Theories. Heuristics and Hypotheses in Sciences. Cham: Springer.

Kant, I. (1992). Lectures on Logic. Cambridge: Cambridge University Press.

Kapitan, T. (1992). Peirce and the Autonomy of Abductive Reasoning. Erkenntnis, 37(1): 1-26.

Laplace, P.S. (1951). A Philosophical Essay on Probabilities. New York: Dover Publications. [1 ${ }^{\text {st }}$ French ed.: 1814].

Laudan, L. (1981). A Confutation of Convergent Realism. Philosophy of Science, 48(1): 19-49.

Lipton, P. (2004). Inference to the Best Explanation. London, Routledge. [1 ${ }^{\text {st }}$ ed.: 1991].

Longo, G. (2012). Incomputability in Physics and Biology. Mathematical Structures in Computer Science, 22(5): 880-900.

Magnus, P.D. (2006). What's New about the New Induction? Synthese, 148(2): 295-301.

Magnus, P.D. (2010). Inductions, Red Herrings, and the Best Explanation for the Mixed Record of Science. The British Journal for the Philosophy of Science, 61(4): 803-819. 
Nickles, T. (1980). Introductory Essay: Scientific Discovery and the Future of Philosophy of Science. In: Nickles, T. (ed.), Scientific Discovery, Logic, and Rationality. Dordrecht: D. Reidel Publishing Company: 1-59.

Nickles, T. (2009). The Strange Story of Scientific Method. In: Meheus, J., Nickles, T. (eds.), Models of Discovery and Creativity. Dordrecht: Springer: 167-207.

Pollock, J.L. (1983). Epistemology and Probability. Synthese, 55(2): 231-252.

Pólya, G. (1941). Heuristic Reasoning and the Theory of Probability. The American Mathematical Monthly, 48(7): 450-465.

Popper, K.R. (2005). The Logic of Scientific Discovery. London: Routledge.

Putnam, H. (1975). Mathematics, Matter and Method. Philosophical Papers. Volume 1. Cambridge: Cambridge University Press.

Reed, B. (2011). Certainty. In: Zalta, E.N. (ed.), The Stanford Encyclopedia of Philosophy, URL $=\langle$ https://plato.stanford.edu/archives/win2011/entries/certainty/ $>$.

Rigo-Lemini, M., Martínez-Navarro, B. (2017). Epistemic States of Convincement. A Conceptualization from the Practice of Mathematicians and Neurobiology. In: Xolocotzin, U.E. (ed.), Understanding Emotions in Mathematical Thinking and Learning. London: Academic Press: 97-131.

Rowbottom, D.P. (2016). Extending the Argument from Unconceived Alternatives: Observations, Models, Predictions, Explanations, Methods, Instruments, Experiments, and Values. Synthese, DOI:10.1007/s11229-016-1132-y.

Ruhmkorff, S. (2011). Some Difficulties for the Problem of Unconceived Alternatives. Philosophy of Science, 78(5): 875-886.

Saatsi, J., Psillos, S., Winther, R.G., Stanford, K. (2009). Grasping at Realist Straws. Metascience, 18 (3): 370-379.

Salmon, W.C. (1990). The Appraisal of Theories: Kuhn Meets Bayes. Proceedings of the Biennial Meeting of the Philosophy of Science Association, 2: 325-332.

Schickore, J. (2014). Scientific Discovery. In: Zalta, E.N. (ed.), The Stanford Encyclopedia of Philosophy, URL = <http://plato.stanford.edu/archives/spr2014/entries/scientificdiscovery/>.

Schupbach, J.N. (2011). Studies in the Logic of Explanatory Power. PhD thesis, University of Pittsburgh, Department of History and Philosophy of Science, Pittsburgh.

Sklar, L., (1981). Do Unborn Hypotheses Have Rights? Pacific Philosophical Quarterly, 62(1): 17-29.

Stanford, P.K. (2006). Exceeding our grasp: Science, history, and the problem of unconceived alternatives. New York: Oxford University Press. 
PENULTIMATE DRAFT - PLEASE CITE THE PUBLISHED VERSION

Topoi. An International Review of Philosophy, DOI: 10.1007/s11245-018-9551-7.

Teira, D. (2011). Frequentist vs. Bayesian Clinical Trials. In: Gifford, F. (ed.), Handbook of the Philosophy of Science. Volume 16. Philosophy of Medicine. Amsterdam: Elsevier: 255-297.

Tulodziecki, D. (2012). Epistemic Equivalence and Epistemic Incapacitation. The British Journal for the Philosophy of Science, 63(2): 313-328.

van Fraassen, B.C. (1989). Laws and Symmetry. Oxford: Oxford University Press.

Vineberg, S. (2016). Dutch Book Arguments. In: Zalta, E.N. (ed.), The Stanford Encyclopedia of Philosophy,

URL $<$ https://plato.stanford.edu/archives/spr2016/entries/dutch-book/>. 